Kragujevac Journal of Mathematics

Volume 44(2) (2020), Pages 251-272.

\title{
LAPLACE TRANSFORM AND HOMOTOPY PERTURBATION METHODS FOR SOLVING THE PSEUDOHYPERBOLIC INTEGRODIFFERENTIAL PROBLEMS WITH PURELY INTEGRAL CONDITIONS
}

\author{
A. NECIB ${ }^{1}$ AND A. MERAD ${ }^{1}$
}

\begin{abstract}
In this paper we defined and investigated the various properties of a class of pseudohyperbolic equation defined on purely integral (nonlocal) conditions. We proved the uniqueness and the existence of the solution using energy inequality (A priori estimates). We found a semi analytical solution using the Laplace transform and Stehfest algorithm method. Next, we used another method called the Homotopy perturbation. Finally, we give some examples for illustration.
\end{abstract}

\section{INTRODUCTION}

Some problems of modern physics and technology can be effectively described in terms of nonlocal (integral) conditions. These nonlocal conditions raise mainly when the data on the boundary cannot be measured directly. The presence of integral terms in the boundary conditions can, greatly, complicate the application of standard numerical techniques such as finite difference procedures, finite elements methods, spectral techniques,..., endless. So far, not much seems to be done for obtaining an explicit solution of heat and wave equations. However, the solvability of these equations has been theoretically studied in terms of existence and uniqueness of a solution. There are two tools used in this paper. The first one is Laplace transformation and the use of its inversed transformation to obtain the numerical solution. The Laplace transformation method has been used to approximate the solution of different classes of linear partial differential equations. Suying et al [25], established

Key words and phrases. Pseudohyperbolic integrodifferential equation, a priori estimate, Laplace transform method, Stehfest algorithm, homotopy perturbation method.

2010 Mathematics Subject Classification. Primary: 05C38, 15A15. Secondary: 05A15, 15A18.

DOI 10.46793/KgJMat2002.251N

Received: October 13, 2017.

Accepted: April 04, 2018. 
a numerical method based on the Laplace transformation for solving initial problem nonlinear dynamic differential equations. The main difficulty in using the Laplace transformation method consists in finding its inverse, because the inverse of transformation is very complex in few situations. To overcome this difficulty, there are many numerical techniques available to invert Laplace transformation. In this work, we focus exclusively on the STEHFEST algorithm [26] in order to efficiently and accurately invert the Laplace transformation (which cannot be done analytically). The application of Lapalce transformation on the equations reduces the problem to a second order inhomogeneous ordinary differential equations with nonlocal conditions. The reduced problem can be solved by the method of variation of parameters. After discretization, the numerical method for inverting the Laplace transformation is used to get an approximation of solution. The second tool is the homotopy perturbation method (HPM), which has been developed by scientists and engineers, in nonlinear problems. This method reduces difficulties of the problem under study into a simple problem which is easy to solve. Most perturbation methods assume the existence of a small parameter, but most nonlinear problems have no small parameter at all. Recently, the application of homotopy perturbation theory has appeared in the work of many scientists $[2,3,7-10,12-18,23]$, which has become a powerful mathematical tool. Recently, S. Abbasbandy [2] applied this method on functional integral equations. The aim of this paper is to establish the existence, uniqueness and the continuous dependence of the data with the solution of second order pseudohyperbolic integrodifferential equations with nonlocal conditions $[5,6]$. The proofs are based on a priori estimates and a combination of Laplace transformation technique with Stehfest algorithm. Furthermore, some examples are given to compare between the approximate and the exact solutions. Later, another semi-analytical technique called Homotopy perturbation method is used. Some testing examples are given to show the efficiency of this method.

\section{Statement of the Problem}

In the rectangular domain $Q=\Omega \times I=\{(x, t): 0<x<1,0<t \leq T\}$, we consider a pseudohyperbolic integrodifferential equation:

$$
\mathcal{L} v=\frac{\partial^{2} v}{\partial t^{2}}-\alpha \frac{\partial^{2} v}{\partial x^{2}}-\beta \frac{\partial^{3} v}{\partial t \partial x^{2}}+\gamma v-\int_{0}^{t} a(t-s) v(x, s) d s=g(x, t),
$$

subject to the initial conditions

$$
\begin{aligned}
& \ell v=v(x, 0)=\Phi(x), \quad 0<x<1, \\
& q v=v_{t}(x, 0)=\Psi(x), \quad 0<x<1,
\end{aligned}
$$

and the purely integral conditions

$$
\int_{0}^{1} v(x, t) d x=n(t), \quad 0<t \leq T
$$




$$
\int_{0}^{1} x v(x, t) d x=m(t), \quad 0<t \leq T,
$$

where $g, \Phi, \Psi, a, n$, and $m$ are known functions, $\alpha, \beta, \gamma$ and $T$ are known positive constants.

\section{Reformulation of the Problem}

Since the integral boundary conditions are inhomogeneous, it is convenient to convert the problem (2.1)-(2.5) to an equivalent problem with homogeneous integral conditions. For this, we introduce a new function $u(x, t)$ as follow:

$$
v(x, t)=u(x, t)+U(x, t),
$$

where

$$
U(x, t)=(-6 x+4) n(t)+(12 x-6) m(t) .
$$

Problem (2.1)-(2.5) with inhomogeneous integral conditions (2.4)-(2.5) can be equivalently reduced to the problem of finding a function $u$ satisfying:

$$
\mathcal{L} u=\frac{\partial^{2} u}{\partial t^{2}}-\alpha \frac{\partial^{2} u}{\partial x^{2}}-\beta \frac{\partial^{3} u}{\partial t \partial x^{2}}+\gamma u-\int_{0}^{t} a(t-s) u(x, s) d s=f(x, t),
$$

with the initial conditions

$$
\begin{aligned}
& \ell u=u(x, 0)=\varphi(x), \quad 0<x<1, \\
& q u=u_{t}(x, 0)=\psi(x), \quad 0<x<1
\end{aligned}
$$

and the purely nonlocal conditions

$$
\begin{array}{cc}
\int_{0}^{1} u(x, t) d x=0, & 0<t \leq T, \\
\int_{0}^{1} x u(x, t) d x=0, & 0<t \leq T,
\end{array}
$$

where

$$
f(x, t)=g(x, t)-\mathcal{L} U(x, t)
$$

and

$$
\begin{aligned}
& \varphi(x)=\Phi(x)-\ell U(x, t), \\
& \psi(x)=\Psi(x)-q U(x, t) .
\end{aligned}
$$

Hence, instead of looking for $v$, we simply look for $u$. The solution of problem (2.1)-(2.5) will be obtained by the relations (3.1)-(3.2). 


\section{A Priori Estimates and its Consequences}

The solution of the problem (3.3)-(3.7) can be considered as a solution of the problem in the operational form:

$$
L u=\mathscr{F},
$$

where $L=(\mathcal{L}, \ell, q)$ is considered from $B$ to $F$, where $B$ is the Banach space of the functions $u \in L^{2}(Q)$, whose norm is:

$$
\|u\|_{B}=\left(\sup _{0 \leqslant \tau \leqslant T} \int_{0}^{1}\left(\left\|\Im_{x} \frac{\partial u}{\partial t}(x, \tau)\right\|^{2}+\left\|\Im_{x} u(x, \tau)\right\|^{2}\right) d x\right)^{\frac{1}{2}},
$$

which is finite, and $F$ is the Hilbert space consisting of all the elements $\mathscr{F}=(f, \varphi, \psi)$ whose norm is:

$$
\|\mathscr{F}\|_{F}=\left(\int_{Q_{\tau}}\|f\|^{2} d x d t+\int_{0}^{1}\left(\|\psi(x)\|^{2}+\|\varphi(x)\|^{2}\right) d x\right)^{\frac{1}{2}},
$$

which is finite.

The domain $D(L)$ of the operator $L$ is the set of all the functions $u$ such that $\frac{\partial u}{\partial x}, \frac{\partial^{2} u}{\partial t^{2}}, \frac{\partial u}{\partial x}, \frac{\partial^{2} u}{\partial x^{2}}, \frac{\partial^{3} u}{\partial t \partial x^{2}} \in L^{2}(Q)$ and $u$ satisfies (3.6) as well as (3.7).

First an a priori estimates is established. Then, the uniqueness and continuous dependence of the solution with respect to the data are immediately conveyed.

Theorem 4.1. If $u(x, t)$ is a solution of problem (3.3)-(3.7) and $|a(t)| \leq a_{1}, 0 \leq t \leq$ $T$ and $\beta$ satisfying the condition $\beta \geqslant \frac{T^{4} a_{1} \varepsilon_{1}^{2}+a_{1}}{8 \varepsilon_{1}}+\frac{1}{8 \varepsilon_{2}}, f \in C(\bar{Q})$, then

$$
\|u\|_{B} \leq C\|\mathscr{F}\|_{F},
$$

where $C$ is a positive constant independent of $u, u \in D(L)$ and

$$
C=\left(\frac{\max \left\{\frac{1}{2}, \varepsilon_{2}, \frac{1}{2} \gamma+\alpha\right\}}{\min \{1, \gamma+2 \alpha\}}\right)^{\frac{1}{2}}
$$

Proof. We put $\Im_{x} u=\int_{0}^{x} u(\xi, t) d \xi$ and $\Im_{x}^{2} u=\int_{0}^{x} \int_{0}^{\eta} u(\xi, t) d \xi d \eta$. Multiplying the equation (3.3) by the integro differential operator $M u=-\Im_{x}^{2} \frac{\partial u}{\partial t}$ and integrating on the subdomain $Q_{\tau}=(0,1) \times(0, \tau)$, where $0 \leq \tau \leq T$, we obtain:

$$
\begin{aligned}
& -\int_{Q_{\tau}} \frac{\partial^{2} u}{\partial t^{2}} \cdot \Im_{x}^{2}\left(\frac{\partial u}{\partial t}\right) d x d t+\alpha \int_{Q_{\tau}} \frac{\partial^{2} u}{\partial x^{2}} \cdot \Im_{x}^{2}\left(\frac{\partial u}{\partial t}\right) d x d t+\beta \int_{Q_{\tau}} \frac{\partial^{3} u}{\partial t \partial x^{2}} \cdot \Im_{x}^{2}\left(\frac{\partial u}{\partial t}\right) d x d t \\
& +\beta \int_{Q_{\tau}} \frac{\partial^{3} u}{\partial t \partial x^{2}} \cdot \Im_{x}^{2}\left(\frac{\partial u}{\partial t}\right) d x d t-\gamma \int_{Q_{\tau}} u \cdot \Im_{x}^{2}\left(\frac{\partial u}{\partial t}\right) d x d t \\
= & -\int_{Q_{\tau}}\left(\int_{0}^{t} a(t-s) u(x, s) d s\right) \cdot \Im_{x}^{2}\left(\frac{\partial u}{\partial t}\right) d x d t-\int_{Q_{\tau}} f \cdot \Im_{x}^{2}\left(\frac{\partial u}{\partial t}\right) d x d t .
\end{aligned}
$$

The integration by parts of each term on the left-hand side of the equation (4.1) gives:

$$
-\int_{Q_{\tau}} \frac{\partial^{2} u}{\partial t^{2}} \cdot \Im_{x}^{2} \frac{\partial u}{\partial t} d x d t=\frac{1}{2} \int_{0}^{1}\left\|\Im_{x} \frac{\partial u}{\partial t}(x, \tau)\right\|^{2} d x-\frac{1}{2} \int_{0}^{1}\left\|\Im_{x} \psi(x)\right\|^{2} d x,
$$




$$
\alpha \int_{Q_{\tau}} \frac{\partial^{2} u}{\partial x^{2}} \cdot \Im_{x}^{2} \frac{\partial u}{\partial t} d x d t=\frac{1}{2} \alpha \int_{0}^{1}\|u(x, \tau)\|^{2} d x-\frac{1}{2} \alpha \int_{0}^{1}\|\varphi(x)\|^{2} d x
$$

$\beta \int_{Q_{\tau}} \frac{\partial^{3} u}{\partial t \partial x^{2}} \cdot \Im_{x}^{2} \frac{\partial u}{\partial t} d x d t=\beta \int_{Q_{\tau}}\left\|\frac{\partial u}{\partial t}\right\|^{2} d x d t$

$$
-\gamma \int_{Q_{\tau}} u \cdot \Im_{x}^{2} \frac{\partial u}{\partial t} d x d t=\frac{1}{2} \gamma \int_{0}^{1}\left\|\Im_{x} u(x, \tau)\right\|^{2} d x-\frac{1}{2} \gamma \int_{0}^{1}\left\|\Im_{x} \varphi(x)\right\|^{2} d x .
$$

The substitution of (4.2), (4.3), (4.4) and (4.5) into (4.1) gives:

$$
\begin{aligned}
& \frac{1}{2} \int_{0}^{1}\left\|\Im_{x} \frac{\partial u}{\partial t}(x, \tau)\right\|^{2} d x+\frac{1}{2} \gamma \int_{0}^{1}\left\|\Im_{x} u(x, \tau)\right\|^{2} d x \\
& +\frac{1}{2} \alpha \int_{0}^{1}\|u(x, \tau)\|^{2} d x+\beta \int_{Q_{\tau}}\left\|\frac{\partial u}{\partial t}\right\|^{2} d x d t \\
= & \frac{1}{2} \int_{0}^{1}\left\|\Im_{x} \psi(x)\right\|^{2} d x+\frac{1}{2} \gamma \int_{0}^{1}\left\|\Im_{x} \varphi(x)\right\|^{2} d x+\frac{1}{2} \alpha \int_{0}^{1}\|\varphi(x)\|^{2} d x \\
& -\int_{Q_{\tau}} f \cdot \Im_{x}^{2} \frac{\partial u}{\partial t} d x d t-\int_{Q_{\tau}}\left(\int_{0}^{t} a(t-s) u(x, s) d s\right) \cdot \Im_{x}^{2} \frac{\partial u}{\partial t} d x d t .
\end{aligned}
$$

Using Poincare's inequality type

$$
\begin{aligned}
\int_{0}^{1}\left\|\Im_{x}^{2} u(x, \tau)\right\|^{2} d x & \leq \frac{1}{2} \int_{0}^{1} \Im_{x}\|u(x, \tau)\|^{2} d x, \\
\int_{0}^{1}\left\|\Im_{x} u(x, \tau)\right\|^{2} d x & \leq \frac{1}{2} \int_{0}^{1}\|u(x, \tau)\|^{2} d x, \\
\int_{Q_{\tau}}\left\|\int_{0}^{t} u(x, s) d s\right\|^{2} d x d t & \leq \frac{T^{2}}{2} \int_{Q_{\tau}}\|u\|^{2} d x d t,
\end{aligned}
$$

we obtain:

$$
\begin{aligned}
& \frac{1}{2} \int_{0}^{1}\left\|\Im_{x} \frac{\partial u}{\partial t}(x, \tau)\right\|^{2} d x+\left(\frac{1}{2} \gamma+\alpha\right) \int_{0}^{1} \Im_{x}\|u(x, \tau)\|^{2} d x+\beta \int_{Q_{\tau}}\left\|\frac{\partial u}{\partial t}\right\|^{2} d x d t \\
\leq & \frac{1}{4} \int_{0}^{1}\|\psi(x)\|^{2} d x+\left(\frac{1}{4} \gamma+\frac{1}{2} \alpha\right) \int_{0}^{1}\|\varphi(x)\|^{2} d x-\int_{Q_{\tau}} f \cdot \Im_{x}^{2} \frac{\partial u}{\partial t} d x d t \\
& -\int_{Q_{\tau}}\left(\int_{0}^{t} a(t-s) u(x, s) d s\right) \cdot \Im_{x}^{2} \frac{\partial u}{\partial t} d x d t .
\end{aligned}
$$

Using the Cauchy inequality with $\varepsilon$, the right-hand side of (4.1) is bounded

$$
\begin{aligned}
& \int_{Q_{\tau}} f \cdot \Im_{x}^{2} \frac{\partial u}{\partial t} d x d t-\int_{Q_{\tau}}\left(\int_{0}^{t} a(t-s) u(x, s) d s\right) \cdot \Im_{x}^{2} \frac{\partial u}{\partial t} d x d t \\
\leqslant & \frac{\varepsilon_{2}}{2} \int_{Q_{\tau}}\|f\|^{2} d x d t+\frac{1}{8 \varepsilon_{2}} \int_{Q_{\tau}}\left\|\frac{\partial u}{\partial t}\right\|^{2} d x d t+\frac{a_{1} \varepsilon_{1}}{2} \int_{Q_{\tau}}\left\|\int_{0}^{t} u(x, s) d s\right\|^{2} d x d t \\
& +\frac{a_{1}}{2 \varepsilon_{1}} \int_{Q_{\tau}}\left\|\Im_{x}^{2} \frac{\partial u}{\partial t}\right\|^{2} d x d t
\end{aligned}
$$


we get

$$
\begin{aligned}
& \int_{Q_{\tau}} f \cdot \Im_{x}^{2} \frac{\partial u}{\partial t} d x d t-\int_{Q_{\tau}}\left(\int_{0}^{t} a(t-s) u(x, s) d s\right) \cdot \Im_{x}^{2} \frac{\partial u}{\partial t} d x d t \\
\leq & \frac{\varepsilon_{2}}{2} \int_{Q_{\tau}}\|f\|^{2} d x d t+\left(\frac{T^{4} a_{1} \varepsilon_{1}^{2}+a_{1}}{8 \varepsilon_{1}}+\frac{1}{8 \varepsilon_{2}}\right) \int_{Q_{\tau}}\left\|\frac{\partial u}{\partial t}\right\|^{2} d x d t,
\end{aligned}
$$

using (4.8) into (4.7) we obtain:

$$
\begin{aligned}
& \int_{0}^{1}\left\|\Im_{x} \frac{\partial u}{\partial t}(x, \tau)\right\|^{2} d x+(\gamma+2 \alpha) \int_{0}^{1}\left\|\Im_{x} u(x, \tau)\right\|^{2} d x \\
& +2\left(\beta-\frac{T^{4} a_{1} \varepsilon_{1}^{2}+a_{1}}{8 \varepsilon_{1}}-\frac{1}{8 \varepsilon_{2}}\right) \int_{Q_{\tau}}\left\|\frac{\partial u}{\partial t}\right\|^{2} d x d t \\
& \leq \varepsilon_{2} \int_{Q_{\tau}}\|f\|^{2} d x d t+\frac{1}{2} \int_{0}^{1}\|\psi(x)\|^{2} d x+\left(\frac{1}{2} \gamma+\alpha\right) \int_{0}^{1}\|\varphi(x)\|^{2} d x,
\end{aligned}
$$

if $\beta$ satisfies the condition $\beta \geqslant \frac{T^{4} a_{1} \varepsilon_{1}^{2}+a_{1}}{8 \varepsilon_{1}}+\frac{1}{8 \varepsilon_{2}}$ we obtain:

$$
\begin{aligned}
& \int_{0}^{1}\left\|\Im_{x} \frac{\partial u}{\partial t}(x, \tau)\right\|^{2} d x+(\gamma+2 \alpha) \int_{0}^{1}\left\|\Im_{x} u(x, \tau)\right\|^{2} d x \\
\leq & \varepsilon_{2} \int_{Q_{\tau}}\|f\|^{2} d x d t+\frac{1}{2} \int_{0}^{1}\|\psi(x)\|^{2} d x+\left(\frac{1}{2} \gamma+\alpha\right) \int_{0}^{1}\|\varphi(x)\|^{2} d x .
\end{aligned}
$$

Since the right-hand side of (4.9) is independent of $\tau$, we take the supremum with respect to $\tau$ from 0 to $T$ in the left-hand side we obtain:

$$
\begin{gathered}
\sup _{0 \leqslant \tau \leqslant T}\left\{\int_{0}^{1}\left\|\Im_{x} \frac{\partial u}{\partial t}(x, \tau)\right\|^{2} d x+\int_{0}^{1}\left\|\Im_{x} u(x, \tau)\right\|^{2} d x\right\} \\
\leqslant C\left(\int_{Q_{\tau}}\|f\|^{2} d x d t+\int_{0}^{1}\|\psi(x)\| d x+\int_{0}^{1}\|\varphi(x)\|^{2} d x\right) .
\end{gathered}
$$

We thus obtain inequality

$$
\|u\|_{B} \leq C\|\mathscr{F}\|_{F}
$$

with

$$
C=\left(\frac{\max \left\{\frac{1}{2}, \varepsilon_{2}, \frac{1}{2} \gamma+\alpha\right\}}{\min \{1, \gamma+2 \alpha\}}\right)^{\frac{1}{2}}
$$

Corollary 4.1. If problem (3.3)-(3.7) has a solution, then this solution is unique and depends continuously on $(f, \varphi, \psi)$.

\section{The Existence of Solution}

Theorem 5.1. If $\beta$ satisfies the condition $\beta \geq \frac{T^{4} a_{1} \varepsilon_{1}^{2}+a_{1}}{8 \varepsilon_{1}}+\frac{1}{8 \varepsilon_{2}}$, then the problem (3.3)-(3.7) admits a unique strong solution $u=\bar{L}^{-1}(f, \varphi, \psi)=\overline{L^{-1}}(f, \varphi, \psi)$. 
Proof. To prove that the problem (3.3)-(3.7) admits a strong solution for any arbitrary $(f, \varphi, \psi) \in F$, it is sufficient to prove that $R(L)$ is dense in $F$, first of all, for the case where $L$ is reduced to $L_{0}=(\mathcal{L}, \ell, q)$ where its domain is $D\left(L_{0}\right)=$ $\{u / u \in D(L): \ell u=0$ and $q u=0\}$. For this purpose, we demonstrate the following proposition.

Proposition 5.1. Under the conditions of Theorem 5.1, for $\omega \in L^{2}(Q)$ and for all $u \in D\left(L_{0}\right)$, we have

$$
\int_{Q} \mathcal{L} u \cdot \omega d x d t=0
$$

then $\omega$ renders null almost everywhere in $Q$.

Proof. The equality (5.1) can be written as follows

$$
\begin{aligned}
\int_{Q_{\tau}} \frac{\partial^{2} u}{\partial t^{2}} \cdot \omega d x d t= & \alpha \int_{Q_{\tau}} \frac{\partial^{2} u}{\partial x^{2}} \cdot \omega d x d t+\beta \int_{Q_{\tau}} \frac{\partial^{3} u}{\partial t \partial x^{2}} \cdot \omega d x d t-\gamma \int_{Q_{\tau}} u \cdot \omega d x d t \\
& +\int_{Q_{\tau}}\left(\int_{0}^{t} a(t-s) u(x, s) d s\right) \cdot \omega d x d t
\end{aligned}
$$

From the equality (5.2), we give the function $\omega$ in terms of $u$ as follows:

$$
\omega=-\Im_{x}^{2} \frac{\partial u}{\partial t} .
$$

By substituting $\omega$ in (5.2) by its representation (5.3), we obtain:

$$
\begin{aligned}
& \int_{Q_{\tau}} \frac{\partial^{2} u}{\partial t^{2}} \cdot\left(-\Im_{x}^{2} \frac{\partial u}{\partial t}\right) d x d t=\alpha \int_{Q_{\tau}} \frac{\partial^{2} u}{\partial x^{2}} \cdot\left(-\Im_{x}^{2} \frac{\partial u}{\partial t}\right) d x d t \\
& +\beta \int_{Q_{\tau}} \frac{\partial^{3} u}{\partial t \partial x^{2}} \cdot\left(-\Im_{x}^{2} \frac{\partial u}{\partial t}\right) d x d t-\gamma \int_{Q_{\tau}} u \cdot\left(-\Im_{x}^{2} \frac{\partial u}{\partial t}\right) d x d t \\
& +\int_{Q_{\tau}}\left(\int_{0}^{t} a(t-s) u(x, s) d s\right) \cdot\left(-\Im_{x}^{2} \frac{\partial u}{\partial t}\right) d x d t,
\end{aligned}
$$

Integrating by parts and taking into account conditions (3.6) and (3.7), we obtain:

$$
\begin{aligned}
\int_{Q_{\tau}} \frac{\partial^{2} u}{\partial t^{2}}\left(-\Im_{x}^{2} \frac{\partial u}{\partial t}\right) d x d t & =\frac{1}{2} \int_{0}^{1}\left\|\Im_{x} \frac{\partial u}{\partial t}(x, \tau)\right\|^{2} d x-\frac{1}{2} \int_{0}^{1}\left\|\Im_{x} q u\right\|^{2} d x \\
& =\frac{1}{2} \int_{0}^{1}\left\|\Im_{x} \frac{\partial u}{\partial t}(x, \tau)\right\|^{2} d x,
\end{aligned}
$$

$$
\begin{aligned}
\alpha \int_{Q_{\tau}} \frac{\partial^{2} u}{\partial x^{2}} \cdot\left(-\Im_{x}^{2} \frac{\partial u}{\partial t}\right) d x d t & =-\frac{1}{2} \alpha \int_{0}^{1}\|u(x, \tau)\|^{2} d x+\frac{1}{2} \alpha \int_{0}^{1}\|\ell u\|^{2} d x \\
& =-\frac{1}{2} \alpha \int_{0}^{1}\|u(x, \tau)\|^{2} d x
\end{aligned}
$$

$$
\beta \int_{Q_{\tau}} \frac{\partial^{3} u}{\partial t \partial x^{2}} \cdot\left(-\Im_{x}^{2} \frac{\partial u}{\partial t}\right) d x d t=-\beta \int_{Q_{\tau}}\left\|\frac{\partial u}{\partial t}\right\|^{2} d x d t
$$




$$
\begin{aligned}
-\gamma \int_{Q_{\tau}} u \cdot\left(-\Im_{x}^{2} \frac{\partial u}{\partial t}\right) d x d t & =-\frac{1}{2} \gamma \int_{0}^{1}\left\|\Im_{x} u(x, \tau)\right\|^{2} d x+\frac{1}{2} \gamma \int_{0}^{1}\left\|\Im_{x} \ell u\right\|^{2} d x \\
& =-\frac{1}{2} \gamma \int_{0}^{1}\left\|\Im_{x} u(x, \tau)\right\|^{2} d x .
\end{aligned}
$$

By substituting (5.5), (5.6), (5.7) and (5.8) into (5.4) we obtain:

$$
\begin{aligned}
& \quad \frac{1}{2} \int_{0}^{1}\left\|\Im_{x} \frac{\partial u}{\partial t}(x, \tau)\right\|^{2} d x+\beta \int_{Q_{\tau}}\left\|\frac{\partial u}{\partial t}\right\|^{2} d x d t \\
& +\frac{1}{2} \gamma \int_{0}^{1}\left\|\Im_{x} u(x, \tau)\right\|^{2} d x+\frac{1}{2} \alpha \int_{0}^{1}\|u(x, \tau)\|^{2} d x \\
& \leq \int_{Q_{\tau}}\left(\int_{0}^{t} a(t-s) u(x, s) d s\right) \cdot\left(-\Im_{x}^{2} \frac{\partial u}{\partial t}\right) d x d t .
\end{aligned}
$$

By the use of Cauchy inequality with $\varepsilon$, the right-hand side of (5.9) is bounded

$$
\int_{Q_{\tau}}\left(\int_{0}^{t} a(t-s) u(x, s) d s\right) \cdot\left(-\Im_{x}^{2} \frac{\partial u}{\partial t}\right) d x d t \leq \frac{T^{4} a_{1} \varepsilon_{1}^{2}+a_{1}}{8 \varepsilon_{1}} \int_{Q_{\tau}}\left\|\frac{\partial u}{\partial t}\right\|^{2} d x d t .
$$

By using (5.10) into (5.9) we obtain:

$$
\begin{aligned}
& \frac{1}{2} \int_{0}^{1}\left\|\Im_{x} \frac{\partial u}{\partial t}(x, \tau)\right\|^{2} d x+\left(\beta-\frac{T^{4} a_{1} \varepsilon_{1}^{2}+a_{1}}{8 \varepsilon_{1}}\right) \int_{Q_{\tau}}\left\|\frac{\partial u}{\partial t}\right\|^{2} d x d t \\
& +\frac{1}{2} \gamma \int_{0}^{1}\left\|\Im_{x} u(x, \tau)\right\|^{2} d x+\frac{1}{2} \alpha \int_{0}^{1}\|u(x, \tau)\|^{2} d x \leq 0,
\end{aligned}
$$

if $\beta$ satisfies the condition $\beta \geq \frac{T^{4} a_{1} \varepsilon_{1}^{2}+a_{1}}{8 \varepsilon_{1}}+\frac{1}{8 \varepsilon_{2}}$ we obtain:

$$
\frac{1}{2} \int_{0}^{1}\left\|\Im_{x} \frac{\partial u}{\partial t}(x, \tau)\right\|^{2} d x+\frac{1}{2} \gamma \int_{0}^{1}\left\|\Im_{x} u(x, \tau)\right\|^{2} d x+\frac{1}{2} \alpha \int_{0}^{1}\|u(x, \tau)\|^{2} d x \leq 0 .
$$

Since the right-hand side of (5.11) is independent from $\tau$. We take the supremum, with respect to $\tau$ from 0 to $T$, in the left-hand side we obtain:

$$
\sup _{0 \leqslant \tau \leqslant T}\left\{\frac{1}{2} \int_{0}^{1}\left\|\Im_{x} \frac{\partial u}{\partial t}(x, \tau)\right\|^{2} d x+\frac{1}{2} \gamma \int_{0}^{1}\left\|\Im_{x} u(x, \tau)\right\|^{2} d x+\frac{1}{2} \alpha \int_{0}^{1}\|u(x, \tau)\|^{2} d x\right\} \leq 0 .
$$

We get $u=0$. Now, we put $u=0$ in (5.3), which gives $\omega \equiv 0$ in $L^{2}(Q)$.

\section{Laplace Transform Method and Stehfest Algorithm}

6.1. Laplace transform method. Laplace transform is an efficient method for solving many differential equations and partial differential equations. The main difficulty with Laplace transform method, is inverting the domain of Laplace solution into the real domain (see $[4,20-22]$ ). In this section we shall apply the Laplace transform technique to find solutions of partial differential equations.

Supposing that $v(x, t)$ is defined and is of exponential order for $t \geq 0$, i.e., it exists $A, \gamma>0$ and $t_{0}>0$ such that $|v(x, t)| \leq A \exp (\gamma t)$ for $t \geq t_{0}$. Then the Laplace 
transform $V(x, s)$, exists and is given by

$$
V(x, s)=\mathscr{L}\{v(x, t): t \rightarrow s\}=\int_{0}^{\infty} v(x, t) \exp (-s t) d t,
$$

where $s$ is a positive real parameter. Taking the Laplace transform on both sides of (2.1), we get

$(6.1)-(\alpha+s \beta) \frac{\partial^{2} V}{\partial x^{2}}(x, s)+\left(s^{2}+\gamma-A(s)\right) V(x, s)=G(x, s)+\psi(x)+s \varphi(x)-\beta \varphi^{\prime \prime}(x)$,

where $G(x, s)=\mathscr{L}\{g(x, t) ; t \rightarrow s\}$ and $A(s)=\mathscr{L}\{a(t) ; t \rightarrow s\}$.

Similarly, we have

$$
\begin{aligned}
\int_{0}^{1} V(x, s) d x & =N(s), \\
\int_{0}^{1} x V(x, s) d x & =M(s),
\end{aligned}
$$

where

$$
\begin{aligned}
& N(s)=\mathscr{L}\{n(t): t \rightarrow s\}, \\
& M(s)=\mathscr{L}\{m(t): t \rightarrow s\} .
\end{aligned}
$$

Thus, the considered equation is reduced into a boundary-value problem governed by a second-order inhomogeneous ordinary differential equation.

Now, we distinguish the following cases.

Case 1. If $A(s)=s^{2}+\gamma$, we obtain a general solution of (6.1) as follows

$$
\begin{aligned}
V(x, s)= & \frac{-1}{(\alpha+s \beta)} \int_{0}^{x}(x-\tau)\left[G(\tau, s)+\psi(\tau)+s \varphi(\tau)-\beta \varphi^{\prime \prime}(\tau)\right] d \tau \\
& +C_{1}(s) x+C_{2}(s)
\end{aligned}
$$

where $C_{1}$ and $C_{2}$ are arbitrary functions of $s$. By substituting (6.4) into (6.2) and (6.3), we get

$$
\begin{aligned}
C_{1}= & \frac{1}{(\alpha+s \beta)} \int_{0}^{1}\left(G(\tau, s)+\psi(\tau)+s \varphi(\tau)-\beta \varphi^{\prime \prime}(\tau)\right)\left(2 \tau^{3}-3 \tau^{2}+1\right) d \tau \\
& +12 M(s)-6 N(s) \\
C_{2}= & \frac{-2}{(\alpha+s \beta)} \int_{0}^{1}\left(G(\tau, s)+\psi(\tau)+s \varphi(\tau)-\beta \varphi^{\prime \prime}(\tau)\right)\left(-\tau^{3}+2 \tau^{2}-\tau\right) d \tau \\
& -6 M(s)+4 N(s) .
\end{aligned}
$$

In general it is impossible to evaluate the integrals in (6.4)-(6.6) exactly. So one may have to resort to numerical integration in order to compute them, for example, the Gauss's formula (25.4.30) given in Abramowitz and Stegun [1] may be employed to calculate these integrals numerically, we have the following approximations for the 
integrals:

(6.7)

$$
\begin{aligned}
& \int_{0}^{1}\left(G(\tau, s)+\psi(\tau)+s \varphi(\tau)-\beta \varphi^{\prime \prime}(\tau)\right)\left(2 \tau^{3}-3 \tau^{2}+1\right) d \tau \\
\simeq & \frac{1}{2} \sum_{i=1}^{N} w_{i}\left[G\left(\frac{1}{2}\left[x_{i}+1\right] ; s\right)+\psi\left(\frac{1}{2}\left[x_{i}+1\right]\right)+s \varphi\left(\frac{1}{2}\left[x_{i}+1\right]\right)-\beta \varphi^{\prime \prime}\left(\frac{1}{2}\left[x_{i}+1\right]\right)\right] \\
& \times \frac{1}{4}\left(x_{i}-1\right)^{2}\left(x_{i}+2\right),
\end{aligned}
$$

$$
\begin{aligned}
& \int_{0}^{1}\left(G(\tau, s)+\psi(\tau)+s \varphi(\tau)-\beta \varphi^{\prime \prime}(\tau)\right)\left(-\tau^{3}+2 \tau^{2}-\tau\right) d \tau \\
\simeq & \frac{1}{2} \sum_{i=1}^{N} w_{i}\left[G\left(\frac{1}{2}\left[x_{i}+1\right] ; s\right)+\psi\left(\frac{1}{2}\left[x_{i}+1\right]\right)+s \varphi\left(\frac{1}{2}\left[x_{i}+1\right]\right)-\beta \varphi^{\prime \prime}\left(\frac{1}{2}\left[x_{i}+1\right]\right)\right] \\
& \times \frac{1}{8}\left[-x_{i}^{3}+x_{i}^{2}+x_{i}-5\right], \\
& \int_{0}^{x}(x-\tau)\left[G(\tau, s)+\psi(\tau)+s \varphi(\tau)-\beta \varphi^{\prime \prime}(\tau)\right] d \tau
\end{aligned}
$$

$$
\simeq \frac{x}{2} \sum_{i=1}^{N} w_{i}\left[G\left(\frac{x}{2}\left[x_{i}+1\right] ; s\right)+\psi\left(\frac{x}{2}\left[x_{i}+1\right]\right)+s \varphi\left(\frac{x}{2}\left[x_{i}+1\right]\right)-\beta \varphi^{\prime \prime}\left(\frac{x}{2}\left[x_{i}+1\right]\right)\right] .
$$

Case 2. If $A(s)<s^{2}+\gamma$, we obtain a general solution of (6.1) as follows

$$
V(x, s)=\frac{-1}{R(\alpha+s \beta)} \int_{0}^{x}\left(G(\tau, s)+\psi(\tau)+s \varphi(\tau)-\beta \varphi^{\prime \prime}(\tau)\right) \sinh R(x-\tau) d \tau
$$

where

$$
R=\sqrt{\frac{s^{2}+\gamma-A(s)}{\alpha+s \beta}}
$$

and $C_{1}, C_{2}$ are arbitrary functions of $s$. By substituting (6.10) into (6.2) and (6.3), we get

$$
\begin{aligned}
& \left(e^{R}-1\right) C_{1}+\left(1-e^{-R}\right) C_{2} \\
= & \frac{1}{R(\alpha+s \beta)} \int_{0}^{1}\left(G(\tau, s)+\psi(\tau)+s \varphi(\tau)-\beta \varphi^{\prime \prime}(\tau)\right)(\cosh R(1-\tau)-1) d \tau+R N(s) \\
& \times\left[(R-1) e^{R}+1\right] C_{1}+\left[-(R+1) e^{R}+1\right] C_{2} \\
= & \frac{1}{R(\alpha+s \beta)} \int_{0}^{1}\left(G(\tau, s)+\psi(\tau)+s \varphi(\tau)-\beta \varphi^{\prime \prime}(\tau)\right) \\
& \times[R(\cosh R(1-\tau)-\tau)-\sinh R(1-\tau)] d \tau+R^{2} M(s),
\end{aligned}
$$


where

$$
\left(\begin{array}{l}
C_{1}(s) \\
C_{2}(s)
\end{array}\right)=\left(\begin{array}{ll}
a_{11}(s) & a_{12}(s) \\
a_{21}(s) & a_{22}(s)
\end{array}\right)^{-1} \times\left(\begin{array}{l}
b_{1}(s) \\
b_{2}(s)
\end{array}\right),
$$

and

$$
\begin{aligned}
a_{11}(s)= & \left(e^{R}-1\right), \\
a_{12}(s)= & \left(1-e^{-R}\right), \\
a_{21}(s)= & (R-1) e^{R}+1 \\
a_{22}(s)= & -(R+1) e^{R}+1, \\
b_{1}(s)= & \frac{1}{R(\alpha+s \beta)} \int_{0}^{1}\left(G(\tau, s)+\psi(\tau)+s \varphi(\tau)-\beta \varphi^{\prime \prime}(\tau)\right)(\cosh R(1-\tau)-1) d \tau \\
& +R N(s), \\
b_{2}(s)= & \frac{1}{R(\alpha+s \beta)} \int_{0}^{1}\left(G(\tau, s)+\psi(\tau)+s \varphi(\tau)-\beta \varphi^{\prime \prime}(\tau)\right) \\
& \times[R(\cosh R(1-\tau)-\tau)-\sinh R(1-\tau)] d \tau+R^{2} M(s) .
\end{aligned}
$$

We have the following approximations for the integrals:

$$
\begin{aligned}
& \int_{0}^{1}\left(G(\tau, s)+\psi(\tau)+s \varphi(\tau)-\beta \varphi^{\prime \prime}(\tau)\right)(\cosh R(1-\tau)-1) d \tau \\
\simeq & \frac{1}{2} \sum_{i=1}^{N} w_{i}\left[G\left(\frac{1}{2}\left[x_{i}+1\right] ; s\right)+\psi\left(\frac{1}{2}\left[x_{i}+1\right]\right)+s \varphi\left(\frac{1}{2}\left[x_{i}+1\right]\right)-\beta \varphi^{\prime \prime}\left(\frac{1}{2}\left[x_{i}+1\right]\right)\right] \\
& \times\left(\cosh R\left(1-\frac{1}{2}\left[x_{i}+1\right]\right)-1\right),
\end{aligned}
$$

$$
\begin{aligned}
& \int_{0}^{1}\left(G(\tau, s)+\psi(\tau)+s \varphi(\tau)-\beta \varphi^{\prime \prime}(\tau)\right)[R(\cosh R(1-\tau)-\tau)-\sinh R(1-\tau)] d \tau \\
\simeq & \frac{1}{2} \sum_{i=1}^{N} w_{i}\left[G\left(\frac{1}{2}\left[x_{i}+1\right] ; s\right)+\psi\left(\frac{1}{2}\left[x_{i}+1\right]\right)+s \varphi\left(\frac{1}{2}\left[x_{i}+1\right]\right)-\beta \varphi^{\prime \prime}\left(\frac{1}{2}\left[x_{i}+1\right]\right)\right] \\
& \times\left[R\left(\cosh R\left(1-\frac{1}{2}\left[x_{i}+1\right]\right)-\frac{1}{2}\left[x_{i}+1\right]\right)-\sinh R\left(1-\frac{1}{2}\left[x_{i}+1\right]\right)\right],
\end{aligned}
$$

$$
\begin{aligned}
& \int_{0}^{x}\left[G(\tau, s)+\psi(\tau)+s \varphi(\tau)-\beta \varphi^{\prime \prime}(\tau)\right] \sinh R(x-\tau) d \tau \\
\simeq & \frac{x}{2} \sum_{i=1}^{N} w_{i}\left[G\left(\frac{x}{2}\left[x_{i}+1\right] ; s\right)+\psi\left(\frac{x}{2}\left[x_{i}+1\right]\right)+s \varphi\left(\frac{x}{2}\left[x_{i}+1\right]\right)-\beta \varphi^{\prime \prime}\left(\frac{x}{2}\left[x_{i}+1\right]\right)\right] \\
& +\sinh R\left(x-\frac{x}{2}\left[x_{i}+1\right]\right) .
\end{aligned}
$$


Case 3. If $A(s)>s^{2}+\gamma$, we obtain a general solution of (6.1) as follows

$$
\begin{aligned}
V(x, s)= & \frac{-1}{R(\alpha+s \beta)} \int_{0}^{x}\left[G(\tau, s)+\psi(\tau)+s \varphi(\tau)-\beta \varphi^{\prime \prime}(\tau)\right] \sin R(x-\tau) d \tau \\
& +C_{1}(s) \cos R x+C_{2}(s) \sin R x
\end{aligned}
$$

where

$$
R=\sqrt{\frac{s^{2}+\gamma-A(s)}{\alpha+s \beta}}
$$

and $C_{1}, C_{2}$ are arbitrary functions of $s$. By substituting (6.14) into (6.2) and (6.3), we get

$$
\begin{aligned}
& \sin R C_{1}+(1-\cos R) C_{2} \\
= & \frac{1}{R(\alpha+s \beta)} \int_{0}^{1}\left(G(\tau, s)+\psi(\tau)+s \varphi(\tau)-\beta \varphi^{\prime \prime}(\tau)\right)(-\cos R(1-\tau)+1) d \tau+R N(s) \\
& \times(R \sin R+\cos R-1) C_{1}+(-R \cos R+\sin R) C_{2} \\
= & \frac{1}{R(\alpha+s \beta)} \\
& \times \int_{0}^{1}\left(G(\tau, s)+\psi(\tau)+s \varphi(\tau)-\beta \varphi^{\prime \prime}(\tau)\right)(R \cos R(1-\tau)+R \tau+\sin R(1-\tau)) d \tau \\
& +R^{2} M(s),
\end{aligned}
$$

where

and

$$
\left(\begin{array}{l}
C_{1}(s) \\
C_{2}(s)
\end{array}\right)=\left(\begin{array}{ll}
a_{11}(s) & a_{12}(s) \\
a_{21}(s) & a_{22}(s)
\end{array}\right)^{-1} \times\left(\begin{array}{c}
b_{1}(s) \\
b_{2}(s)
\end{array}\right)
$$

$$
\begin{aligned}
a_{11}(s)= & \sin R, \\
a_{12}(s)= & (1-\cos R), \\
a_{21}(s)= & R \sin R+\cos R-1), \\
a_{22}(s)= & (-R \cos R+\sin R), \\
b_{1}(s)= & \frac{1}{R(\alpha+s \beta)} \int_{0}^{1}\left(G(\tau, s)+\psi(\tau)+s \varphi(\tau)-\beta \varphi^{\prime \prime}(\tau)\right)(-\cos R(1-\tau)+1) d \tau \\
& +R N(s), \\
b_{2}(s)= & \frac{1}{R(\alpha+s \beta)} \int_{0}^{1}\left(G(\tau, s)+\psi(\tau)+s \varphi(\tau)-\beta \varphi^{\prime \prime}(\tau)\right) \\
& \times(R \cos R(1-\tau)+R \tau+\sin R(1-\tau)) d \tau+R^{2} M(s) .
\end{aligned}
$$

We have the following approximations for the integrals:

$$
\int_{0}^{1}\left(G(\tau, s)+\psi(\tau)+s \varphi(\tau)-\beta \varphi^{\prime \prime}(\tau)\right)(-\cos R(1-\tau)+1) d \tau
$$




$$
\begin{aligned}
\simeq & \frac{1}{2} \sum_{i=1}^{N} w_{i}\left[G\left(\frac{1}{2}\left[x_{i}+1\right] ; s\right)+\psi\left(\frac{1}{2}\left[x_{i}+1\right]\right)+s \varphi\left(\frac{1}{2}\left[x_{i}+1\right]\right)-\beta \varphi^{\prime \prime}\left(\frac{1}{2}\left[x_{i}+1\right]\right)\right] \\
& \times\left(-\cos R\left(1-\frac{1}{2}\left[x_{i}+1\right]\right)+1\right)
\end{aligned}
$$

$$
\begin{aligned}
& \int_{0}^{1}\left(G(\tau, s)+\psi(\tau)+s \varphi(\tau)-\beta \varphi^{\prime \prime}(\tau)\right)(R \cos R(1-\tau)+R \tau+\sin R(1-\tau)) d \tau \\
\simeq & \frac{1}{2} \sum_{i=1}^{N} w_{i}\left[G\left(\frac{1}{2}\left[x_{i}+1\right] ; s\right)+\psi\left(\frac{1}{2}\left[x_{i}+1\right]\right)+s \varphi\left(\frac{1}{2}\left[x_{i}+1\right]\right)-\beta \varphi^{\prime \prime}\left(\frac{1}{2}\left[x_{i}+1\right]\right)\right] \\
& \times\left[R \cos R\left(1-\frac{1}{2}\left[x_{i}+1\right]\right)+\frac{R}{2}\left[x_{i}+1\right]+\sin R\left(1-\frac{1}{2}\left[x_{i}+1\right]\right)\right],
\end{aligned}
$$

$$
\begin{aligned}
& \int_{0}^{x}\left[G(\tau, s)+\psi(\tau)+s \varphi(\tau)-\beta \varphi^{\prime \prime}(\tau)\right] \sin R(x-\tau) d \tau \\
\simeq & \frac{x}{2} \sum_{i=1}^{N} w_{i}\left[G\left(\frac{x}{2}\left[x_{i}+1\right] ; s\right)+\psi\left(\frac{x}{2}\left[x_{i}+1\right]\right)+s \varphi\left(\frac{x}{2}\left[x_{i}+1\right]\right)-\beta \varphi^{\prime \prime}\left(\frac{x}{2}\left[x_{i}+1\right]\right)\right] \\
& \times \sin R\left(x-\frac{x}{2}\left[x_{i}+1\right]\right),
\end{aligned}
$$

where $x_{i}$ and $w_{i}$ the abscissa and weights, are defined as

$$
x_{i}: i^{\text {th }} \text { zero of } P_{n}(x), \quad w_{i}=\frac{2}{\left(1-x_{i}^{2}\right)}\left(P_{n}^{\prime}(x)\right)^{2} .
$$

Their tabulated values can be found in [1] for different values of $N$.

\section{The Stehfest Algorithm (Numerical Inversion of Laplace TRANSFORM)}

Sometimes, an analytical inversion of a domain of Laplace solution is difficult to obtain; therefore a numerical inversion method must be used. A nice comparison of four frequently used numerical Laplace inversion algorithms is given by H. Hassanzadeh et al [19]. In this work we use the Stehfest's algorithm [26] that is easy to implement. This numerical technique was first introduced by Graver [11] and its algorithm then offered by [26]. Stehfest's algorithm approximates the time domain solution as follows

$$
v(x, t) \approx \frac{\ln 2}{t} \sum_{n=1}^{2 m} \beta_{n} V\left(x ; \frac{n \ln 2}{t}\right),
$$

where $m$ is a positive integer,

$$
\beta_{n}=(-1)^{n+m} \sum_{k=\left[\frac{n+1}{2}\right]}^{\min \{n, m\}} \frac{k^{m}(2 k) !}{(m-k) ! k !(k-1) !(n-k) !(2 k-n) !},
$$

and $[q]$ denotes the integer part of the real number $q$. 
7.1. Numerical examples. In this section, we report some results of numerical computations using Laplace transformation method proposed in the previous section. These techniques are applied to solve the problem defined by (2.1)-(2.5) for particular functions $g, \Phi, \Psi, n, m$ and positive constants $\alpha, \beta$ and $\gamma$. The method of solution is easily implemented on the computer, used Matlab 7.9.3 program. The numerical results are obtained by $N=8$ in (6.7)-(6.9), (6.11)-(6.13), (6.15)-(6.17) and $m=5$ in (7.1)-(7.2). Then, we compared the exact solution with numerical solution. An excellent agreement was found between the two of them.

Example 7.1. We take

$$
\begin{aligned}
g(x, t) & =-e^{-x} \sinh (t), a(t)=0, \quad 0<x<1,0<t \leq T \text { and } \alpha=1, \beta=1, \gamma=0, \\
\Phi(x) & =\exp (-x), \quad 0<x<1, \\
\Psi(x) & =0, \quad 0<x<1, \\
n(t) & =\left(1-e^{-1}\right) \cosh (t), \quad 0<t \leq T, \\
m(t) & =\left(1-2 e^{-1}\right) \cosh (t), \quad 0<t \leq T .
\end{aligned}
$$

In this case, the exact solution given by

$$
v(x, t)=e^{-x} \cosh (t), \quad 0<x<1,0<t \leq T .
$$

For $t=0.10, x \in[0.10,0.90]$, we calculate $v$ numerically using the proposed method of solution and compare it with the exact solution in Table 1.

TABLE 1.

\begin{tabular}{|l|l|l|l|l|l|}
\hline$x$ & 0.10 & 0.30 & 0.50 & 0.70 & 0.90 \\
\hline$v$ exact & 0.9093654 & 0.7445254 & 0.6095658 & 0.490703 & 0.4086042 \\
\hline$v$ numerical & 0.9093851 & 0.7443921 & 0.6097452 & 0.500183 & 0.4080919 \\
\hline Relative error & 0.000217 & -0.0001790 & 0.0002943 & 0.0022295 & -0.0012538 \\
\hline
\end{tabular}

Example 7.2. We take

$$
\begin{aligned}
g(x, t) & =\left(3 e^{-t}-1\right) \cos (2 \pi x), a(t)=1, \quad 0<x<1,0<t \leq T \text { and } \alpha=\beta=\gamma=1, \\
\Phi(x) & =\cos (2 \pi x), \quad 0<x<1, \\
\Psi(x) & =-\cos (2 \pi x), \quad 0<x<1, \\
n(t) & =0, \quad 0<t \leq T, \\
m(t) & =0, \quad 0<t \leq T .
\end{aligned}
$$

In this case, the exact solution given by

$$
v(x, t)=e^{-t} \cos (2 \pi x), \quad 0<x<1,0<t \leq T .
$$

For $t=0.1, x \in[0.1,0.9]$, we calculate $v$ numerically using the proposed method of solution and compare it with the exact solution in Table 2. 


\section{TABLE 2.}

\begin{tabular}{|l|l|l|l|l|l|}
\hline$x$ & 0.10 & 0.30 & 0.50 & 0.70 & 0.90 \\
\hline$v$ exact & 0.7320288 & -0.2796101 & -0.9048374 & -0.2796101 & 0.7320288 \\
\hline$v$ numerical & 0.7324162 & -0.2795921 & -0.9047562 & -0.2795421 & 0.7321329 \\
\hline Relative error & 0.0005292 & -0.0000644 & -0.0000897 & -0.0002432 & 0.0001422 \\
\hline
\end{tabular}

Example 7.3. We take

$$
\begin{aligned}
g(x, t) & =-e^{x} \sinh t, a(t)=0, \quad 0<x<1,0<t \leq T \text { and } \alpha=1, \beta=1, \gamma=0, \\
\Phi(x) & =\exp (x), \quad 0<x<1 \\
\Psi(x) & =0, \quad 0<x<1 \\
n(t) & =(e-1) \cosh (t), \quad 0<t \leq T \\
m(t) & =\cosh (t), \quad 0<t \leq T .
\end{aligned}
$$

In this case, the exact solution given by

$$
v(x, t)=e^{x} \cosh (t), \quad 0<x<1,0<t \leq T .
$$

For $t=0.1, x \in[0.1,0.9]$, we calculate $v$ numerically using the proposed method of solution and compare it with the exact solution in Table 3.

TABLE 3.

\begin{tabular}{|l|l|l|l|l|l|}
\hline$x$ & 0.10 & 0.30 & 0.50 & 0.70 & 0.90 \\
\hline$v$ exact & 1.1107014 & 1.3566137 & 1.6569717 & 2.0238299 & 2.4719114 \\
\hline$v$ numerical & 1.1106841 & 1.3565926 & 1.6569459 & 2.0237984 & 2.4718729 \\
\hline Relative error & 0.0000155 & 0.0000155 & 0.0000155 & 0.0000155 & 0.0000155 \\
\hline
\end{tabular}

Example 7.4. We take

$$
\begin{aligned}
g(x, t) & =0, a(t)=0, \quad 0<x<1, \quad 0<t \leq T \text { and } \alpha=\frac{1}{2}, \beta=\frac{1}{2}, \gamma=0, \\
\Phi(x) & =\exp (x), \quad 0<x<1 \\
\Psi(x) & =\exp (x), \quad 0<x<1 \\
n(t) & =(e-1) \exp (t), \quad 0<t \leq T, \\
m(t) & =\exp (t), \quad 0<t \leq T .
\end{aligned}
$$

In this case, the exact solution given by

$$
v(x, t)=e^{x+t}, \quad 0<x<1,0<t \leq T .
$$

For $t=0.1, x \in[0.1,0.9]$, we calculate $v$ numerically using the proposed method of solution and compare it with the exact solution in Table 4 . 


\section{TABLE 4.}

\begin{tabular}{|l|l|l|l|l|l|}
\hline$x$ & 0.10 & 0.30 & 0.50 & 0.70 & 0.90 \\
\hline$v$ exact & 1.221403 & 1.491825 & 1.822119 & 2.225541 & 2.718281 \\
\hline$v$ numerical & 1.221407 & 1.491830 & 1.822125 & 2.22555 & 2.718291 \\
\hline Relative error & 0.0000031 & 0.0000033 & 0.0000032 & 0.000004 & 0.0000036 \\
\hline
\end{tabular}

\section{Homotopy Perturbation Method with Laplace Transform} (LT- HPM)

8.1. Basic idea of homotopy perturbation method. The homotopy perturbation method was proposed first by He in 1998 [12] and was developed and improved by He [13-17]. To illustrate the basic ideas of this method, we consider the following non-linear functional equation:

$$
A(u)-f(r)=0, \quad r \in \Omega,
$$

with the following boundary condition:

$$
B\left(u ; \frac{\partial u}{\partial \eta}\right)=0, \quad r \in \Gamma
$$

where $A$ is a general functional operator, $B$ a boundary operator, $f(r)$ is a known analytical function and $\Gamma$ is the boundary of the domain. The operator $A$ can be decomposed into two operators $L$ and $N$, where $L$ is linear, and $N$ is nonlinear operator. Equation (8.1) can be, therefore, written as follows:

$$
L(u)+N(u)-f(r)=0 .
$$

Using the homotopy technique, we construct an homotopy:

$$
v(r ; p): \Omega \times[0 ; 1] \rightarrow R,
$$

which satisfies:

$$
H(v ; p)=(1-p)\left[L(v)-L\left(u_{0}\right)\right]+p[A(v)-f(r)]=0, \quad p \in[0,1], r \in \Omega,
$$

or

$$
H(v ; p)=L(v)-L\left(u_{0}\right)+p\left[L\left(u_{0}\right)+N(v)-f(r)\right]=0,
$$

where $p \in[0 ; 1]$ is an embedding parameter, $u_{0}$ is an initial approximation for the solution of equation (8.1), which satisfies the boundary conditions. Obviously, from equations. (8.4) and (8.5) we will have:

$$
\begin{gathered}
H(v ; 0)=L(v)-L\left(u_{0}\right)=0, \\
H(v ; 1)=A(v)-f(r)=0 .
\end{gathered}
$$

The changing values of $p$ from zero to unity are just that of $v(r ; p)$ from $u_{0}(r)$ to $u(r)$.In topology, this is called deformation, and $L(v)-L\left(u_{0}\right), A(v)-f(r)$ are called homotopic. In 1998 J. H. He, used the imbedding parameter $p$ as a "small parameter", 
and assume that the solution of equations (8.4) and (8.5) can be written as a power series in $p$ :

$$
v=v_{0}+p v_{1}+p^{2} v_{2}+\cdots
$$

We take $p \rightarrow 1$, results in the approximation to the solution of equation (8.1),

$$
u=\lim _{p \rightarrow 1} v=v_{0}+v_{1}+v_{2}+\cdots .
$$

The combination of the perturbation method and the homotopy method is called the homotopy perturbation method (HPM), which has eliminated limitations of the traditional perturbation techniques. The series (8.9) is convergent for more cases. Some criteria are suggested for convergence of the Series (8.9), in [12].

8.2. Laplace transform HPM. Taking the Laplace transform of (2.1) we obtain a new partial differential equation (6.1).

According to HPM, for solving equation (6.1) we construct an homotopy by Madani et al [18], as the following form:

$$
\left(s^{2}+\gamma-A(s)\right) V(x, s)=p\left[-(\alpha+s \beta) \frac{\partial^{2} V}{\partial x^{2}}(x, s)\right]+G(x, s)+\psi(x)+s \varphi(x)-\beta \varphi^{\prime \prime}(x) .
$$

Now, let us present the solution of equation (8.10) as the following form:

$$
V(x, s)=\sum_{j=0}^{\infty} p^{j} V_{j}(x, s),
$$

where $v_{j}(x ; s), j=0,1,2, \ldots$ are functions which should be determined. By substituting (8.11) into (8.10), we get

$$
\begin{aligned}
\left(s^{2}+\gamma-A(s)\right) \sum_{j=0}^{\infty} p^{j} V_{j}(x, s)= & p\left[-(\alpha+s \beta) \frac{\partial^{2}}{\partial x^{2}}\left(\sum_{j=0}^{\infty} p^{j} V_{j}(x, s)\right)\right] \\
& +G(x, s)+\psi(x)+s \varphi(x)-\beta \varphi^{\prime \prime}(x) .
\end{aligned}
$$

Equating the coefficients of $p$ with the same powers in (8.12) leads to

$$
\begin{aligned}
p^{0} & :\left(s^{2}+\gamma-A(s)\right) V_{0}(x, s)=G(x, s)+\psi(x)+s \varphi(x)-\beta \varphi^{\prime \prime}(x), \\
p^{1} & :\left(s^{2}+\gamma-A(s)\right) V_{1}(x, s)=(\alpha+s \beta) \frac{\partial^{2}}{\partial x^{2}} V_{0}(x, s), \\
p^{2} & :\left(s^{2}+\gamma-A(s)\right) V_{2}(x, s)=(\alpha+s \beta) \frac{\partial^{2}}{\partial x^{2}} V_{1}(x, s), \\
& \vdots \\
p^{n+1}: & \left(s^{2}+\gamma-A(s)\right) V_{n+1}(x, s)=(\alpha+s \beta) \frac{\partial^{2}}{\partial x^{2}} V_{n}(x, s) .
\end{aligned}
$$


From where

we obtain:

$$
\left\{\begin{array}{l}
V_{n+1}(x, s)=\frac{\alpha+s \beta}{s^{2}+\gamma-A(s)} \frac{\partial^{2}}{\partial x^{2}} V_{n}(x, s), \\
V_{0}(x, s)=\frac{G(x, s)+\psi(x)+s \varphi(x)-\beta \varphi^{\prime \prime}(x)}{s^{2}+\gamma-A(s)}
\end{array}\right.
$$

$$
V_{n}(x, s)=\left(\frac{\alpha+s \beta}{s^{2}+\gamma-A(s)}\right)^{n} \frac{G_{x}^{(2 n)}(x, s)+\psi_{x}^{(2 n)}(x)+s \varphi_{x}^{(2 n)}(x)-\beta \varphi_{x}^{(2 n+2)}(x)}{s^{2}+\gamma-A(s)},
$$

when $p \rightarrow 1$, (8.11) becomes the approximate solution of equation (6.1), i.e.,

$$
V(x ; s) \cong H_{n}(x ; s)=\sum_{j=0}^{n} V_{j}(x, s) .
$$

The physical solution $v(x ; t)$ can be recovered approximately from $H_{n}(x ; s)$ according to the Stehfest's algorithm [26].

Taking the inverse $\mathscr{L}^{-1}$ from both sides of (8.13) we get the approximate solution of $(2.1)-(2.5)$ :

$$
v(x ; t) \cong \mathscr{L}^{-1}\left(H_{n}(x ; s)\right)=\mathscr{L}^{-1}\left(\sum_{j=0}^{n} V_{j}(x, s)\right) .
$$

Example 8.1. We take

$$
\begin{aligned}
g(x, t) & =-e^{x} \sinh t, a(t)=0, \quad 0<x<1,0<t \leq T \text { and } \alpha=1, \beta=1, \gamma=0, \\
\Phi(x) & =\exp (x), \quad 0<x<1, \\
\Psi(x) & =0, \quad 0<x<1, \\
n(t) & =(e-1) \cosh (t), \quad 0<t \leq T, \\
m(t) & =\cosh (t), \quad 0<t \leq T .
\end{aligned}
$$

We obtain

$$
\begin{aligned}
V_{n}(x, s) & =\left(\frac{s+1}{s^{2}}\right)^{n} \frac{s^{2}-s-1}{s^{2}\left(s^{2}-1\right)} e^{x}, \\
V(x ; s) & \cong H_{n}(x ; s)=\sum_{j=0}^{n} V_{j}(x, s)=e^{x}\left[1-\left(\frac{s+1}{s^{2}}\right)^{n+1}\right] \frac{s}{\left(s^{2}-1\right)}, \\
v(x ; t) & \cong e^{x} \mathscr{L}^{-1}\left\{\left[1-\left(\frac{s+1}{s^{2}}\right)^{n+1}\right] \frac{s}{\left(s^{2}-1\right)}\right\},
\end{aligned}
$$

when $n=1, \ldots, 4$ (see Table 5$)$.

The closed form of the series $1+t+\frac{1}{2 !} t^{2}+\frac{1}{3 !} t^{3}+\frac{1}{4 !} t^{4}+\cdots$ is $e^{t}$ which gives an exact solution of the problem $v(x ; t)=e^{x} \cosh t$.

Example 8.2. We take

$$
g(x, t)=0, a(t)=0, \quad 0<x<1,0<t \leq T \text { and } \alpha=\frac{1}{2}, \beta=\frac{1}{2}, \gamma=0,
$$


TABle 5 .

\begin{tabular}{|l|l|}
\hline$n$ & $v(x ; t)$ \\
\hline 1 & $\left(\cosh t+2\left(-e^{t}+1+t+\frac{1}{4} t^{2}\right)\right) e^{x}$ \\
\hline 2 & $\left(\cosh t+4\left(-e^{t}+1+t+\frac{1}{2 !} t^{2}+\frac{1}{8} t^{3}+\frac{1}{96} t^{4}\right)\right) e^{x}$ \\
\hline 3 & $\left(\cosh t+8\left(-e^{t}+1+t+\frac{1}{2 !} t^{2}+\frac{1}{3 !} t^{3}+\frac{7}{192} t^{4}+\frac{1}{240} t^{5}+\frac{1}{5760} t^{6}\right)\right) e^{x}$ \\
\hline 4 & $\left(\cosh t+16\left(-e^{t}+1+t+\frac{1}{2 !} t^{2}+\frac{1}{3 !} t^{3}+\frac{1}{4 !} t^{4}+\frac{1}{128} t^{5}+\frac{11}{11520} t^{6}+\frac{1}{16128} t^{7}+\frac{1}{645120} t^{8}\right)\right) e^{x}$ \\
\hline
\end{tabular}

$$
\begin{aligned}
\Phi(x) & =\exp (x), \quad 0<x<1, \\
\Psi(x) & =\exp (x), \quad 0<x<1, \\
n(t) & =(e-1) e^{t}, \quad 0<t \leq T, \\
m(t) & =e^{t}, \quad 0<t \leq T .
\end{aligned}
$$

We obtain

$$
\begin{aligned}
V_{n}(x, s) & =\left(\frac{s+1}{2 s^{2}}\right)^{n} \frac{2 s+1}{2 s^{2}} e^{x}, \\
V(x ; s) & \cong H_{n}(x ; s)=\sum_{j=0}^{n} V_{j}(x, s)=e^{x}\left(1-\left(\frac{s+1}{2 s^{2}}\right)^{n+1}\right) \frac{2 s+1}{\left(2 s^{2}-s-1\right)}, \\
v(x ; t) & \cong e^{x} \mathscr{L}^{-1}\left\{\left(1-\left(\frac{s+1}{2 s^{2}}\right)^{n+1}\right) \frac{2 s+1}{\left(2 s^{2}-s-1\right)}\right\},
\end{aligned}
$$

when $n=1, \ldots, 4$ (see Table 6 ).

TABLE 6 .

\begin{tabular}{|l|l|}
\hline$n$ & $v(x ; t)$ \\
\hline 1 & $\left(\frac{1}{24} t^{3}+\frac{3}{8} t^{2}+t+1\right) e^{x}$ \\
\hline 2 & $\left(\frac{1}{960} t^{5}+\frac{1}{48} t^{4}+\frac{7}{48} t^{3}+\frac{1}{2 !} t^{2}+t+1\right) e^{x}$ \\
\hline 3 & $\left(\frac{1}{80640} t^{7}+\frac{1}{2304} t^{6}+\frac{11}{1920} t^{5}+\frac{5}{128} t^{4}+\frac{1}{3 !} t^{3}+\frac{1}{2 !} t^{2}+t+1\right) e^{x}$ \\
\hline 4 & $\left(\frac{1}{11612160} t^{9}+\frac{1}{215040} t^{8}+\frac{1}{10080} t^{7}+\frac{13}{11520} t^{6}+\frac{31}{3840} t^{5}+\frac{1}{4 !} t^{4}+\frac{1}{3 !} t^{3}+\frac{1}{2 !} t^{2}+t+1\right) e^{x}$. \\
\hline
\end{tabular}

The closed form of the series $1+t+\frac{1}{2 !} t^{2}+\frac{1}{3 !} t^{3}+\frac{1}{4 !} t^{4}+\cdots$ is $e^{t}$ which gives an exact solution of the problem $v(x ; t)=e^{x+t}$.

Example 8.3. We take

$$
\begin{aligned}
g(x, t)= & \left(t^{2}+2 t+2\right) x^{2}-4 e^{t}, a(t)=t^{2}, \quad 0<x<1,0<t \leq T \\
& \quad \text { and } \alpha=\beta=\gamma=1, \\
\Phi(x)= & x^{2}, \quad 0<x<1, \\
\Psi(x)= & x^{2}, \quad 0<x<1,
\end{aligned}
$$




$$
\begin{aligned}
& n(t)=\frac{1}{3} e^{t}, \quad 0<t \leq T, \\
& m(t)=\frac{1}{4} e^{t}, \quad 0<t \leq T .
\end{aligned}
$$

We obtain

$$
\begin{aligned}
V_{0}(x, s) & =-\frac{1}{(s-1)\left(s^{5}+s^{3}-2\right)}\left(-s^{5} x^{2}+2 s^{4}-s^{3} x^{2}+2 s^{3}+2 x^{2}\right), \\
V_{1}(x, s) & =2 s^{3} \frac{s+1}{\left(s^{5}+s^{3}-2\right)^{2}}\left(s^{4}+s^{3}+2 s^{2}+2 s+2\right), \\
V_{n}(x, s) & =0, \quad n \geq 2, \\
V(x ; s) & =\sum_{i=0}^{i=1} V_{i}(x, s)=\frac{x^{2}}{s-1}, \\
v(x ; t) & =\mathscr{L}^{-1}\left\{\frac{x^{2}}{s-1}\right\}=x^{2} e^{t},
\end{aligned}
$$

which gives an exact solution of the problem $v(x ; t)=x^{2} e^{t}$.

Example 8.4. We take

$$
\begin{aligned}
g(x, t)= & -\frac{1}{6} x^{2}\left(t^{3} x^{2}+3 t^{2} x^{2}+72 t+144\right), a(t)=t, \quad 0<x<1,0<t \leq T \\
& \text { and } \alpha=\beta=1, \gamma=0 \\
\Phi(x)= & x^{4}, \quad 0<x<1 \\
\Psi(x)= & x^{4}, \quad 0<x<1 \\
n(t)= & \frac{1}{5} t+\frac{1}{5}, \quad 0<t \leq T \\
m(t)= & \frac{1}{6} t+\frac{1}{6}, \quad 0<t \leq T \\
V_{0}(x, s)= & \frac{-s^{4} x^{4}+12 s^{3} x^{2}+12 s^{2} x^{2}+x^{4}}{-s^{5}+s^{4}-s^{3}+s^{2}} \\
V_{1}(x, s)= & -\frac{12}{\left(s^{4}-1\right)^{2}}(s+1)^{3}\left(-s^{3} x^{2}+s^{2} x^{2}+2 s^{2}-s x^{2}+x^{2}\right), \\
V_{2}(x, s)= & \frac{s^{2}}{\left(s^{4}-1\right)^{3}}(s+1)^{2}\left(24 s^{5}+24 s^{4}-24 s-24\right), \\
V_{n}(x, s)= & 0, \quad \text { for all } n \geq 3, \\
V(x, s)= & \sum_{i=0}^{i=2} V_{i}=\frac{1}{s^{2}} x^{4}(s+1), \\
v(x ; t)= & \mathscr{L}^{-1}\left\{\frac{1}{s^{2}} x^{4}(s+1)\right\}=x^{4}(t+1),
\end{aligned}
$$

which gives an exact solution of the problem $v(x ; t)=x^{4}(t+1)$. 


\section{REFERENCES}

[1] M. Abramowitz and I. A. Stegun, Handbook of Mathematical Functions, Dover, New York, 1972.

[2] S. Abbasbandy, Numerical solutions of the integral equations: homotopy perturbation method and Adomian's decomposition method, Appl. Math. Comput. 173(2-3) (2006), 493-500.

[3] G. A. Afrouzi, D. D. Ganji, H. Hosseinzadeh and R. A. Talarposhti, Fourth order Volterra integro-differential equations using modied homotopy-perturbation method, Turkish Journal of Mathematics and Computer Science 3(2) (2011), 179-191.

[4] A. Bouziani and A. Merad, The Laplace transform method for one-dimensional hyperbolic equationwith purely integral conditions, Rom. J. Math. Comput. Sci. 3(2) (2013), 191-204.

[5] A. Bouziani and R. Mechri, The Rothe method to a parabolic integro-differential equation with a nonclassical boundary conditions, Int. J. Stoch. Anal. (2010), Article ID 519684, 16 pages, DOI: 10.1155/519684/(2010)

[6] A. Bouziani and M. S. Temsi, On a pseudohyperbolic equation with nonlocal boundary condition, Kobe J. Math. 21 (2004), 15-31.

[7] D. D. Ganji, G. A. Afrouzi, H. Hosseinzadeh and R. A. Talarposhti, Application of Hmotopy perturbation method to the second kind of nonlinear integral equations, Phys. Lett. A 371(1-2) (2007), 20-25.

[8] D. D. Ganji and A. Sadighi, Application of He's homotopy-perturbation method to nonlinear coupled systems of reaction-diffusion equations, Int. J. Nonlinear Sci. Numer. Simul. 7(4) (2006), 411-418.

[9] D. D. Ganji and A. Rajabi, Assessment of homotopy-perturbation and perturbation methods in heat radiation equations, International Communications in Heat and Mass Transfer 33 (2006), 391-400.

[10] D. D. Ganji and M. Rafei, Solitary wave solutions for a generalized Hirota-Satsuma coupled KdV equation by homotopy perturbation method, Phys. Lett. A 356 (2006), $131-137$.

[11] D. P. Graver, Observing stochastic processes and aproximate transform inversion, Oper. Res. 14 (1966), 444-459.

[12] J. H. He, Homotopy perturbation technique, Comput. Methods Appl. Mech. Engrg. 178 (1999), 257-262.

[13] J. H. He, New interpretation of homotopy perturbation method, Internat. J. Modern Phys. B 20 (2006), 2561-2568.

[14] J. H. He, A coupling method of homotopy technique and perturbation technique for nonlinear problems, International Journal of Non-Linear Mechanics 35(1) (2000), $37-43$.

[15] J. H. He, Limit cycle and bifurcation of nonlinear problems, Chaos Solitons Fractals 26 (2005), 827-833.

[16] J. H. He, Homotopy perturbation method for bifurcation of nonlinear problems, Int. J. Nonlinear Sci. Numer. Simul. 6(2) (2005), 207-208.

[17] J. H. He, Application of homotopy perturbation method to nonlinear wave equations, Chaos Solitons Fractals 26 (2005), 695-700.

[18] M. Madani, M. Fathizadeh, Y. Khan and A. Yildrim, On coupling the homotpy perturbation method ans Laplace transformation, Math. Comput. Modelling 53(2011) (1970), 1937-1945. 
[19] H. Hassanzadeh and M. Pooladi-Darvish, Comparision of different numerical Laplace inversion methods for engineering applications, Appl. Math. Comput. 189 (2007), 1966-1981.

[20] A. Merad and A. Bouziani, Laplace transform technique for pseudoparabolic equation with nonlocal conditiond, Transylvanian Journal of Mathematics and Mechanics 5(1) (2013), 59-64.

[21] A. Merad and A. Bouziani, A method of solution of integro-differential parabolic equation with purely integral conditions, in: Advances in Applied Mathematics and Approximation Theory, Springer Proceeding in Mathematics and Statistics 41, New York, 2013.

[22] A. Merad and A. Bouziani, Solvability the telegraph equation with purely integral conditions, TWMS J. Appl. Eng. Math. 3(2) (2013), 117-125.

[23] M. El-Shahed, Application of He's homotopy perturbation method to Volterra integrodifferential equation, Int. J. Nonlinear Sci. Numer. Simul. 6(2) (2005), 163168.

[24] A. D. Shruti, Numerical solution for nonlocal Sobolev-type differential equations, Electron. J. Differential Equations 19 (2010), 75-83.

[25] Z. Suying, Z. Minzhen, D. Zichen and L. Wencheng, Solution of nonlinear dynamic differential equations based on numerical Laplace transform inversion, Appl. Math. Comput. 189 (2007), 79-86.

[26] H. Stehfest, Numerical inversion of the Laplace transform, Communications of the ACM 13 (1970), 47-49.

${ }^{1}$ Department of Mathematics and Informatics,

LARBI BEN M'HIDI UNIVERSITY,

Oum El Bouaghi 04000, Algeria

Email address: abdelhalimnecib@yahoo.fr

Email address: merad_ahcene@yahoo.fr 